\title{
Correction to: Pseudovarieties of Ordered Completely Regular Semigroups
}

\author{
Jorge Almeida@i and Ondřej Klíma
}

\section{Correction to: Results Math (2019) 74:78 https://doi.org/10.1007/s00025-019-0998-7}

The original version of this article unfortunately contained a mistake. The typesetting of mathematical notation was incorrect. The corrected details are given below for your reading.

In "Preliminaries and Notation" section, third paragraph should read as:

A quasiorder $\leq$ on a semigroup $S$ is stable if, for every $a, s, t \in S$, the following condition holds: if $s \leq t$ then $s a \leq t a$ and $a s \leq a t$. By an ordered semigroup, we mean a semigroup $(S, \cdot)$ which is equipped with a stable partial order. Homomorphisms of ordered semigroups are homomorphisms of semigroups which are isotone. Similar to the unordered case, pseudovarieties of ordered semigroups are classes of finite ordered semigroups closed under taking homomorphic images, (ordered) subsemigroups and finite products. For a pseudovariety $\mathrm{V}$ of finite ordered semigroups, we denote by $\mathrm{V}^{d}$ the pseudovariety of all dually ordered semigroups, i.e., $(S, \cdot, \leq) \in \mathrm{V}^{d}$ if and only if $(S, \cdot, \geq) \in \mathrm{V}$. We say that $\mathrm{V}$ is self-dual if $\mathrm{V}=\mathrm{V}^{d}{ }^{1}$. Such a pseudovariety of ordered semigroups is also characterized by the property that $(S, \cdot, \leq) \in \mathrm{V}$ implies $(S, \cdot,=) \in \mathrm{V}$ : the identity mapping is a homomorphism from the ordered semigroup $(S, \cdot,=)$ onto an arbitrary ordered $\operatorname{semigroup}(S, \cdot, \geq)$, while

\footnotetext{
${ }^{1}$ It is perhaps more common to consider in the purely algebraic context the dual of a semigroup $(S, \cdot)$ to be $(S, *)$ where $a * b=b \cdot a$. This is not the notion of duality concerning us here.
}

The original article can be found online at https://doi.org/10.1007/s00025-019-0998-7. 
the diagonal mapping embeds $(S, \cdot,=)$ into the product $(S, \cdot \leq) \times(S, \cdot, \geq)$. If a pseudovariety of semigroups $\mathrm{V}$ is given, then we may consider the (selfdual) pseudovariety consisting of all ordered semigroups which are members of $\mathrm{V}$ equipped with every possible stable partial order. Conversely, if $\mathrm{V}$ is a self-dual pseudovariety of ordered semigroups, then we may consider the pseudovariety consisting of all semigroups $(S, \cdot)$ such that $(S, \cdot,=) \in \mathrm{V}$. These constructions give a one-to-one correspondence between self-dual pseudovarieties of ordered semigroups and pseudovarieties of semigroups. Note that usually the same symbol is used to denote both a pseudovariety of semigroups and the corresponding self-dual pseudovariety of ordered semigroups.

In "Preliminaries and Notation" section, (seventh sentence of the) ninth paragraph should read as:

By a system of relations $\left(\rho_{n}\right)_{n}$, we mean a family of relations indexed by the positive integers such that $\rho_{n}$ is a relation on $\bar{\Omega}_{n} \mathrm{~S}$ for every $n$. We say that the system $\left(\rho_{n}\right)_{n}$ is fully invariant if, for each continuous homomorphism $\varphi: \bar{\Omega}_{n} \mathrm{~S} \rightarrow \bar{\Omega}_{m} \mathrm{~S}$ and $u \rho_{n} v$, we have $\varphi(u) \rho_{m} \varphi(v)$. Additionally, if every relation $\rho_{n}$ in this system is a closed stable quasiorder on $\bar{\Omega}_{n} \mathrm{~S}$, then we call the system $\rho=\left(\rho_{n}\right)_{n}$ a fully invariant system of closed stable quasiorders. It is not clear whether every such system $\rho$ determines a pseudovariety $\mathrm{V}$ such that $\rho=(\rho \vee, n)$ (see [5] for a discussion concerning a related conjecture). To explain what kind of property is potentially missing, first denote $\tilde{\rho}=\left(\widetilde{\rho_{n}}\right)_{n}$, where $\widetilde{\rho_{n}}$ is the equivalence relation corresponding to $\rho_{n}$. Then, for each $n$, we consider $\bar{\Omega}_{n} \mathrm{~S} / \widetilde{\rho_{n}}$, which is a compact ordered semigroup, where the partial order is $\leq_{\rho_{n}}$. If for each $n \geq 1$ the ordered semigroup $\bar{\Omega}_{n} \mathrm{~S} / \widetilde{\rho_{n}}$ is residually finite, then $\rho=\rho_{\mathrm{V}}$, where $\mathrm{V}$ is given as the class of all finite ordered semigroups which are finite quotients of some ordered semigroup $\left(\bar{\Omega}_{n} \mathrm{~S} / \widetilde{\rho_{n}}, \cdot \leq_{\rho_{n}}\right)$. If this property is true then we call $\rho$ a complete system of pseudoinequalities. Note that, for the corresponding pseudovariety $\mathrm{V}$ of ordered semigroups, we may also write $\mathrm{V}=\llbracket \rho \rrbracket$ to mean $\mathrm{V}=\llbracket \rho_{n}: n \geq 1 \rrbracket$. We talk about a complete system of pseudoidentities when $\mathrm{V}$ is a self-dual pseudovariety of ordered semigroups. Notice also that $\mathrm{V}$ is self-dual if and only if each $\rho_{n}$ is symmetric.

In Theorem 4.8, second and third paragraph of proof should read as:

To show that $\gamma \circ \iota$ is the identity mapping, we need to prove that $(\mathrm{V} \cap$ $\mathrm{NB}) \vee(\mathrm{V} \cap \mathrm{G})=\mathrm{V}$ for every $\mathrm{V} \subseteq \mathrm{NOCR}$. This is true if $\mathrm{V} \models x^{\omega} \leq x^{\omega} y^{\omega} x^{\omega}$, by Lemma 4.7. Clearly, one may use the dual version of the lemma if $\mathrm{V} \models$ $x^{\omega} y^{\omega} x^{\omega} \leq x^{\omega}$. So, we may assume that $\mathrm{V}$ does not satisfy any of these two pseudoinequalities. Thus, by Lemma 4.1, we know that $\mathrm{SI} \subseteq \mathrm{V}$. From Theorem 3.8, we get that $\mathrm{V}$ is self-dual. This means that if we consider an arbitrary ordered semigroup $(S, \cdot, \leq) \in \mathrm{V}$, then the pseudovariety $\mathrm{V}$ contains also the ordered semigroup $(S, \cdot,=)$. Since $(S, \cdot, \leq)$ is a homomorphic image of $(S, \cdot,=)$, we may deal only with the case of unordered semigroups.

So, let $(S, \cdot=) \in \mathrm{V}$ be arbitrary. Now, it is possible to modify the proof of Lemma 4.7 in such a way that $H_{e}^{\top}$ is replaced by $H_{e}^{0}$. Since the partial order on 
$S$ is equality, the mapping $\psi_{e}$ is trivially isotone. Moreover, $\mathrm{V}$ contains $\mathrm{SI}$ and, therefore, $H_{e}^{0} \in(\mathrm{V} \cap \mathrm{NB}) \vee(\mathrm{V} \cap \mathrm{G})$. Thus, we conclude that $S \in(\mathrm{V} \cap \mathrm{NB}) \vee(\mathrm{V} \cap \mathrm{G})$. We have proved the required equality $(\mathrm{V} \cap \mathrm{NB}) \vee(\mathrm{V} \cap \mathrm{G})=\mathrm{V}$.

The original article has been corrected.

Jorge Almeida

CMUP, Department of Matemática, Faculdade de Ciências

Universidade do Porto

Rua do Campo Alegre 687

4169-007 Porto

Portugal

e-mail: jalmeida@fc.up.pt

Ondřej Klíma

Department of Mathematics and Statistics

Masaryk University

Kotláŕská 2

61137 Brno

Czech Republic

e-mail: klima@math.muni.cz

Publisher's Note Springer Nature remains neutral with regard to jurisdictional claims in published maps and institutional affiliations. 